\title{
Roof-Collapsed PDMS Mask for Nanochannel Fabrication
}

\author{
Jinyong Lee, Young-keu Yoon, Jungwook Kim, Yoori Kim, and Kyubong Jo* \\ Department of Chemistry and Interdisciplinary Program of Integrated Biotechnology, Sogang University, \\ Seoul 121-742, Korea. *E-mail: jokyubong@sogang.ac.kr \\ Received October 6, 2010, Accepted October 26, 2010
}

Key Words: Unconventional lithography, Roof-collapsed PDMS mask, Nanochannels

Here we present a radically simple approach for nanochannel fabrication without the use of any expensive equipment or any instrument modifications. Our approach consists of consecutive simple photolithographic steps: first, we prepare sub-micron high but very wide PDMS nanoslits by using soft lithography. Second, as a mask we utilize PDMS nanoslits collapsed on the thinly coated photoresist layer. Standard photolithography transfers roof-collapsed PDMS nanochannels to a sub-micron thick photoresist layer. Then, we add a micropattern overlay by using micromoulding in capillary method. Consequently, we fabricate nanochannels embedded in microchannels by combination of simple processes: soft lithography, roof-collapse, and standard photolithography.

Nanochannel is an important component in a microfluidic device for biochemical analytical systems. Dimensions comparable to biomolecules allow nanochannels to confine, manipulate, and visualize single biomolecules. ${ }^{1-2}$ Recently, considerable attention has been directed to nanochannel confined DNA elongation to determine DNA size without separation. ${ }^{3-4}$ In addition, elongated DNA molecules can be utilized as platforms for biochemical studies such as RNA polymerase on DNA backbones. Accordingly, nanochannel promises more comprehensive biochemical analysis platform.

Conventionally, nanochannel fabrication requires expensive equipment such as electron beam (e-beam) and focused ion beam (FIB) lithography which are prohibitively expensive for a single academic lab. Also, these serial techniques are much slower than the parallel approach of projection lithography such as X-ray lithography, which however requires a huge synchrotron facility to generate nanometer wavelengths. Alternatively, nanoimprint lithography emerged as a simple process with high throughput and high resolution over large area. ${ }^{5}$ However, nanometer resolution template patterns are being sold at a very high price. Thus, contemporary conventional nanofabrication technologies are not appropriate to simply test a variety of creative or premature ideas in an academic lab. As alternative solutions, a number of unconventional approaches have been developed for avoiding these financial limitations. ${ }^{6}$

Recently, a simple unconventional approach for the formation of nanochannels has been reported that short and wide elastomeric nanoslits of polydimethylsiloxane (PDMS) collapse to form gutters of sub-micron for nanofluidic device, called roofcollapsed nanochannels. ${ }^{7-8}$ Paradoxically, roof collapse has been an issue for micro-contact printing ${ }^{9}$ and the formation of PDMS nanoslits. ${ }^{2}$ Several papers have been reported for new theories and experimental findings to avoid elastomeric deform- ation. ${ }^{10-11}$ Comparing to other conventional or unconventional nanochannel fabrication, roof collapse is far easier, but this approach has intrinsic limitations to add more sophisticated and complex structures such as multiple layers. ${ }^{7}$

Here we design a novel approach of transferring roof collapsed PDMS nanochannels to solid nanostructures on the photoresist. Figure 1 illustrates our scheme how to use roof collapsed nanochannel pattern as a photolithographic mask. Briefly, we place PDMS mask $(40 \mu \mathrm{m} \times 700 \mathrm{~nm})$ on contact with a thin layer of photoresist, and then roof collapsing occurs on a photoresist layer. As standard lithography procedure, ultraviolet light is exposed onto the PDMS mask to transfer roof collapsed nanochannels to a thin photoresist layer ranged from $100 \mathrm{~nm}$ to $1 \mu \mathrm{m}$ : the height is controlled by mixing ratio of photoresist with thinner.

Figure 2a shows a scanning electron microscope image of nanochannel pattern on a photoresist layer transferred. Interestingly, the cross section of the nanochannel is an asymmetric peak shape, which resembles the cross-section shape of roofcollapsed channels as previously reported. ${ }^{7-8}$ The reason of asymmetry is that a roof collapsed nanochannel has two sides: one side used to be a nanoslit wall and the other side used to be a nanoslit roof. When a roof collapsed, the wall side is relatively steeper and the roof side is leaned with a tail. A microscope

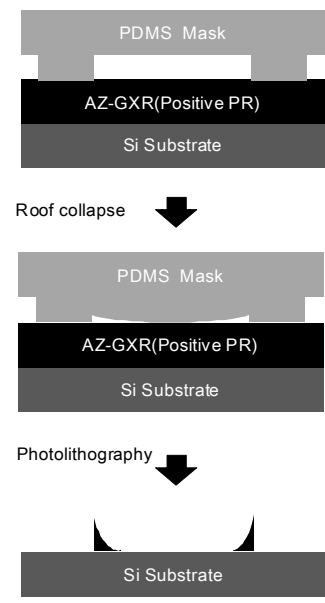

Figure 1. A scheme of nanochannel fabrication with roof collapsed mask. PDMS mask has a nanoslit patterns with the height ranged from 350 to $700 \mathrm{~nm}$ and the widths from $40 \mu \mathrm{m}$ to $80 \mu \mathrm{m}$. PDMS nanoslits are placed on the thin positive photoresis layer of thickness range from $100 \mathrm{~nm}$ to $1 \mu \mathrm{m}$. Then PDMS nanoslits spontaneously collapse. Conventional photolithography aligner transfer roof-collapsed nanochannel patterns to the photoresist layer. 


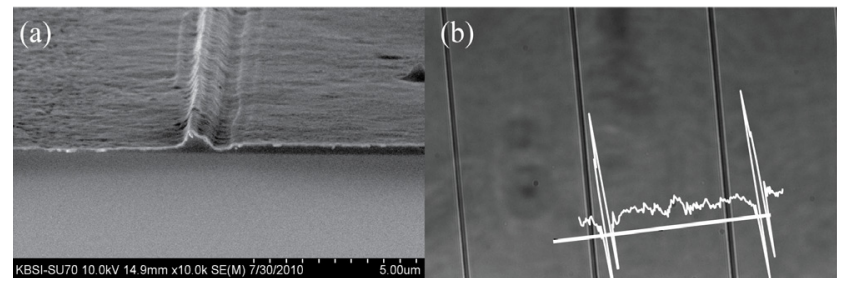

Figure 2. (a) Scanning electron micrograph of fabricated nanochannel's template on silicon wafer. The nanochannel template is utilized for replica molding of PDMS nanochannels. (b) Microscope image for roof collapsed PDMS nanoslits on the glass. Roof collapsed PDMS has dark lines where nanochannels are formed. A darkness profile (white line) shows asymmetic darkness intensity which transferred to a photoresist layer. For better visulaization, the profile is magnified a little with offset. Scale bar $20 \mu \mathrm{m}$.

image of roof collapsed channel explains why roof collapsed PDMS mask transfers peak shape. Figure $2 b$ shows dark lines where roof collapsed PDMS nanochannels formed on the glass surface in this case. Also, intensity profile analysis shows that these dark lines have sharp and asymmetrical peak shapes matching the nanopatterns on photoresist layer.

Pattern transfer can be explained by a couple of related previous studies. The first one is phase shift edge lithography. ${ }^{12}$ The key idea of the phase shift lithography is that PDMS has different index of refraction from that of air, resulting in constructive or destructive interference. This theory explains the edge of solid and perfectly vertical wall with monochromatic light. On the contrary, a roof collapsed PDMS nanochannels has an asymmetric shape. Also, our exposure has broad and multiple wavelengths in the ultraviolet range. The second related theory is light coupling lithography. ${ }^{13}$ The principle is that the light is reflective at the surface roof of pattern, which reduces the light intensity. However, this theory does not explain how asymmetrical sharp peak can be transferred to photoresist layer conserving asymmetrical shapes. Both theories seem to be related to this phenomenon to fully understand this phenomenon.

Transferred nanochannels have a significant advantage over roof-collapsed nanochannels. These nanochannels are solid structures and able to be easily integrated into more complicated microfluidic pattern template, for example, adding a second layer on nanochannels. However, it is not straightforward to add a micropattern overlay using standard photolithography because the nanochannel template layer consists of positive photoresist. Positive photoresist may decompose during the second cycle of photoresist. Thus we utilize another unconventional approach of micromoulding in capillary (MIMIC). ${ }^{14}$ A PDMS micropattern is placed on the surface perfectly adhere by conformal contact. Then the photoresist solution is loaded through a micropattern then polymerized on the $50{ }^{\circ} \mathrm{C}$ hot plate. As a result, we obtain nanochannels embedded in microchannel template, which can be used for PDMS replica molding. Figure 3 shows a PDMS device of microchannels and nanochannels filled with black fountain pen ink that gives off fluorescence. The fluorescent intensity profile shows that there is only a single pixel across the channel which corresponds to $250 \mathrm{~nm}$ width.

Simple and reliable nanochannel fabrication is the key component to develop a nanofluidic analytical system to control biological molecule. Also, as a component, nanochannel should

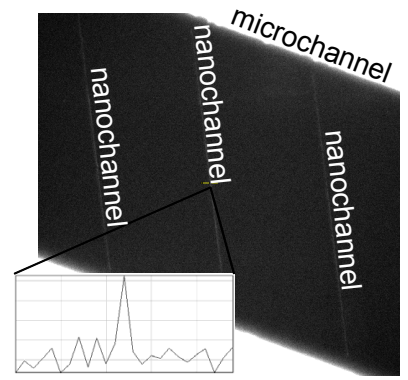

Figure 3. Fluorescent micrograph of ink-filled nanochannels embbed in microchannels. Fluorescent solution is black fountain pen ink diluted with water. The plot profile across the channel shows that fluorescent solution is focused in the range within a single pixel that corresponds to $250 \mathrm{~nm}$.

be able to be integrated into a sophisticated microfluidic device. To meet these both challenge, here we introduce a radically simple but solid approach for nanochannel fabrication without the use of any expensive technology. We utilize roof-collapsed nanochannels as a mask, which allows us to fabricate nanochannels. Then, we add microchannel pattern overlay on the nanochannel pattern by using micromoulding in capillaries. Conclusively, we believe that our simple and inexpensive approach for nanochannel fabrication will provide the basis of testing a variety of creative ideas to control individual biological molecules.

This work is supported by the Sogang University Research Grant No. 200810019.01, and the National Research Foundation of Korea (NRF) grant funded by the Korean Ministry of Education, Science and Technology (MEST) (2010-0015392, 20100028226).

\section{References}

1. Jo, K.; Dhingra, D. M.; Odijk, T.; de Pablo, J. J.; Graham, M. D.; Runnheim, R.; Forrest, D.; Schwartz, D. C. Proc. Nat. Acad. Sci. USA 2007, 104(8), 2673-2678.

2. Lee, J.; Yun, Y. K.; Kim, Y.; Jo, K. Bull. Korean Chem. Soc. 2009, 30(8), 1793-1797.

3. Cao, H.; Yu, Z. N.; Wang, J.; Tegenfeldt, J. O.; Austin, R. H.; Chen, E.; Wu, W.; Chou, S. Y. App. Phys. Lett. 2002, 81(1), 174-176.

4. Tegenfeldt, J. O.; Prinz, C.; Cao, H.; Chou, S.; Reisner, W. W.; Riehn, R.; Wang, Y. M.; Cox, E. C.; Sturm, J. C.; Silberzan, P.; Austin, R. H. Proc. Nat. Acad. Sci. USA 2004, 101(30), 10979-10983.

5. Chou, S. Y.; Krauss, P. R.; Renstrom, P. J. App. Phys. Lett. 1995, 67(21), 3114-3116.

6. Gates, B. D.; Xu, Q. B.; Stewart, M.; Ryan, D.; Willson, C. G.; Whitesides, G. M. Chem. Rev. 2005, 105(4), 1171-1196.

7. Park, S. M.; Huh, Y. S.; Craighead, H. G.; Erickson, D. Proc. Nat. Acad. Sci. USA 2009, 106(37), 15549-15554.

8. Jo, K. Thesis; University of Wisonsin: 2006.

9. Hui, C. Y.; Jagota, A.; Lin, Y. Y.; Kramer, E. J. Langmuir 2002, 18(4), 1394-1407.

10. Huang, Y. G. Y.; Zhou, W. X.; Hsia, K. J.; Menard, E.; Park, J. U.; Rogers, J. A.; Alleyne, A. G. Langmuir 2005, 21(17), 8058-8068.

11. Zhou, W.; Huang, Y.; Menard, E.; Aluru, N. R.; Rogers, J. A.; Alleyne, A. G. App. Phys. Lett. 2005, 87(25), -.

12. Rogers, J. A.; Paul, K. E.; Jackman, R. J.; Whitesides, G. M. App. Phys. Lett. 1997, 70(20), 2658-2660.

13. Schmid, H.; Biebuyck, H.; Michel, B.; Martin, O. J. F.; Piller, N. B. J. Vac. Sci. Tech. B 1998, 16(6), 3422-3425.

14. Kim, E.; Xia, Y. N.; Whitesides, G. M. Nature 1995, 376(6541), 581-584. 